\title{
A Controlled Study on the Characterisation of Bioaerosols Emissions from Compost
}

\author{
Zaheer Ahmad Nasir ${ }^{1, *(\mathbb{D}}$, Catherine Rolph ${ }^{2}$, Samuel Collins ${ }^{3}{ }^{\mathbb{D}}$, David Stevenson ${ }^{4}$, \\ Toni L. Gladding ${ }^{2}{ }^{(}$, Enda Hayes ${ }^{5}$, Ben Williams ${ }^{5}{ }^{\circ}$, Shagun Khera ${ }^{6}$, Simon Jackson ${ }^{6}$, \\ Allan Bennett ${ }^{4}$, Simon Parks ${ }^{4}$, Robert P. Kinnersley ${ }^{7}$, Kerry Walsh ${ }^{7}$, Simon J. T. Pollard ${ }^{1}$, \\ Gillian Drew ${ }^{1}$, Sonia Garcia Alcega ${ }^{1}$, Frederic Coulon ${ }^{1} \oplus$ and Sean Tyrrel ${ }^{1}$ \\ 1 School of Water, Energy and Environment, Cranfield University, Cranfield MK43 0AL, UK; \\ s.pollard@cranfield.ac.uk (S.J.T.P.); g.h.drew@cranfield.ac.uk (G.D.); sg.alcega@gmail.com (S.G.A.); \\ f.coulon@cranfield.ac.uk (F.C.); s.tyrrel@cranfield.ac.uk (S.T.) \\ 2 STEM Faculty, Open University, Walton Hall MK6 7AA, UK; Catherine.Rolph@open.ac.uk (C.R.); \\ T.L.Gladding@open.ac.uk (T.L.G.) \\ 3 Public Health England, Chemical and Environmental Effects Department, Centre for Radiation, \\ Chemical and Environmental Hazards, Oxfordshire OX11 0RQ, UK; samuel.collins@phe.gov.uk \\ 4 Biosafety, Air and Water Microbiology Group, National Infection Service, Public Health England, \\ Salisbury SP4 0JG, UK; David.Stevenson@phe.gov.uk (D.S.); Allan.Bennett@phe.gov.uk (A.B.); \\ Simon.Parks@phe.gov.uk (S.P.) \\ 5 Air Quality Management Resource Centre, Faculty of Environment and Technology, University of the West \\ of England, Bristol BS16 1QY, UK; Enda.Hayes@uwe.ac.uk (E.H.); ben3.williams@uwe.ac.uk (B.W.) \\ 6 School of Biomedical and Healthcare Sciences, Plymouth University, Drake Circus, Plymouth PL4 8AA, UK; \\ shagun.khera@plymouth.ac.uk (S.K.); simon.jackson@plymouth.ac.uk (S.J.) \\ 7 Environment Agency, Research, Assessment and Evaluation, Deanery Road, Bristol BS1 5AH, UK; \\ rob.kinnersley@environment-agency.gov.uk (R.P.K.); kerry.walsh@environment-agency.gov.uk (K.W.) \\ * Correspondence: z.a.nasar@cranfield.ac.uk
}

Received: 22 July 2018; Accepted: 24 September 2018; Published: 28 September 2018

\begin{abstract}
Bioaerosol emissions arising from biowaste treatment are an issue of public concern. To better characterise the bioaerosols, and to assess a range of measurement methods, we aerosolised green waste compost under controlled conditions. Viable and non-viable Andersen samplers, cyclone samplers and a real time bioaerosol detection system (Spectral Intensity Bioaerosol Sensor (SIBS)) were deployed simultaneously. The number-weighted fraction of fluorescent particles was in the range $22-26 \%$ of all particles for low and high emission scenarios. Overall fluorescence spectral profiles seen by the SIBS exhibited several peaks across the 16 wavelength bands from 298 to $735 \mathrm{~nm}$. The size-fractionated endotoxin profile showed most endotoxin resided in the 2.1-9 $\mu \mathrm{m}$ aerodynamic diameter fraction, though up to $27 \%$ was found in a finer size fraction. A range of microorganisms were detected through culture, Matrix Assisted Laser Desorption and Ionisation Time of Flight Mass Spectrometry (MALDI-TOF) and quantitative polymerase chain reaction (qPCR), including Legionella pneumophila serogroup 1. These findings contribute to our knowledge of the physico-chemical and biological characteristics of bioaerosols from composting sites, as well as informing future monitoring approaches and data interpretation for bioaerosol measurement.
\end{abstract}

Keywords: bioaerosols; sampling methods; compost; characterisation

\section{Introduction}

There is public concern as to whether bioaerosol emissions above ambient background levels, which may arise from such sources as waste treatment and agricultural activities, have any health 
implications for nearby communities [1,2]. There is evidence that occupational exposures to high concentrations of bioaerosols can be harmful. The potential health risks from exposure to bioaerosols include infection, toxicity and allergenicity, depending on the bioaerosol composition and on receptor characteristics $[3,4]$. It is less clear what impacts might arise in nearby populations who may be exposed to lower, but still above-background, concentrations. Some evidence is available, for example for composting [3,5], wastewater treatment plants [6-8], and intensive agriculture facilities $[9,10]$. However, such evidence is difficult to gather directly using current methods: results are variable, can be statistically uncertain and tend to rely on proxies for exposure and self-reported impacts. Advancement of bioaerosol risk assessment for industrial processes is limited by uncertainties in hazard characterisation, due to limited health impact data and limitations of current methods for the measurements of bioaerosols [11].

Most available evidence on bioaerosol emissions from regulated processes comes from short duration sampling and culture-based analysis, and may not reflect the complete emissions profile due to time varying site specific factors. The current state of knowledge on emission characteristics is therefore limited, and data on distances at which process-derived bioaerosols remain detectable above ambient background are inconsistent. For example, Pearson et al. [3] identified six studies where Aspergillus fumigatus or total bacteria levels were above the UK Environment Agency's recommended threshold levels more than $250 \mathrm{~m}$ from the site. There are significant knowledge gaps with respect to the composition, magnitude and continuous spatiotemporal characterisation of bioaerosol emissions. Another area of uncertainty is the relationship between particle size and bioaerosol components from an industrial process, and hence their dispersion characteristics, and their association with operations and emission controls. As a result, policies and practices that are based on risk and enable a proportionate response to managing industrial bioaerosol emissions must be made on the basis of incomplete information. Regulators of bioaerosol sources have therefore tended to take a precautionary approach, attempting to ensure that individuals are not exposed to bioaerosol levels higher than those they would typically encounter in the environment.

Improving on the current situation will require better techniques for the rapid detection and characterisation of bioaerosol emissions. Current advancement in bioaerosol detection and characterisation technologies can significantly advance our knowledge on the physical, chemical and biological characteristics of emissions from potential sources. For example, endotoxin is a known pro-inflammatory agent; information on the size distribution of endotoxin-containing aerosols will inform our understanding of endotoxin exposure and inhalation. Improved molecular methods are enabling the identification of pathogenic/allergenic microorganisms in bioaerosols and address the limitations of culture methods, including the detection of viable but non-culturable (VBNC) organisms. Such methods are providing information on bioaerosol characteristics, abundance and diversity but cannot yet provide continuous information to determine temporal variability [12]. In contrast, single particle fluorescence spectroscopy has shown potential to detect and quantify (though not comprehensively speciate) bioaerosol in real-time, and sensors based on this technology are now being deployed in a range of environments including composting facilities. For example O'Connor et al. [13] were able to show that using a WIBS-4 (Waveband Integrated Bioaerosol Sensor model 4, University of Hertfordshire, Hatfield, UK) at a green waste composting site can provide real-time data on bioaerosol release profiles.

Field measurements of bioaerosols are dependent on site activity, meteorology and can be limited by issues of access and confounded by other nearby bioaerosol sources and variations in ambient background bioaerosol levels. Studies under controlled conditions can help characterise the critical characteristics of bioaerosol emissions from a given source. For example the dustiness of organic waste, focusing on endotoxin and culturable microbial components, has been investigated using a rotating drum in the laboratory [14-16]. However, there are very limited data giving detailed characterisation of complex sources of bioaerosols. This study aimed to test a suite of methods to better elucidate 
physico-chemical and biological characteristics of bioaerosols emissions by using them concurrently under controlled conditions, using compost as an example of a bioaerosol emission source.

\section{Materials and Methods}

\subsection{Test Chamber}

The characterisation of bioaerosol emissions from commercial compost was carried out in a controlled test chamber (Figure 1). The walls and ceiling are constructed from panels, faced with white, food-safe, unplasticised Polyvinyl Chloride (UPVC) laminated, zinc coated sheet steel. The test chamber had a volume of $20 \mathrm{~m}^{3}$ and was supplied with a horizontal flow of clean air from a bank of high efficiency particulate air (HEPA) filters, positioned behind a diffuser wall. These filters fed a combination of fresh air and recirculated HEPA filtered air from the chamber. A separate extract filter mounted centrally within the ceiling, drew a small fraction of air to maintain negative pressure and allow for the introduction of fresh air. The chamber is designed to allow the operation of equipment and services remotely from an anteroom, allowing for procedures to be undertaken without staff being present in the test space. The chamber is designed to achieve up to 250 air changes per hour, providing very high levels of air cleanliness and rapid removal of test aerosols.

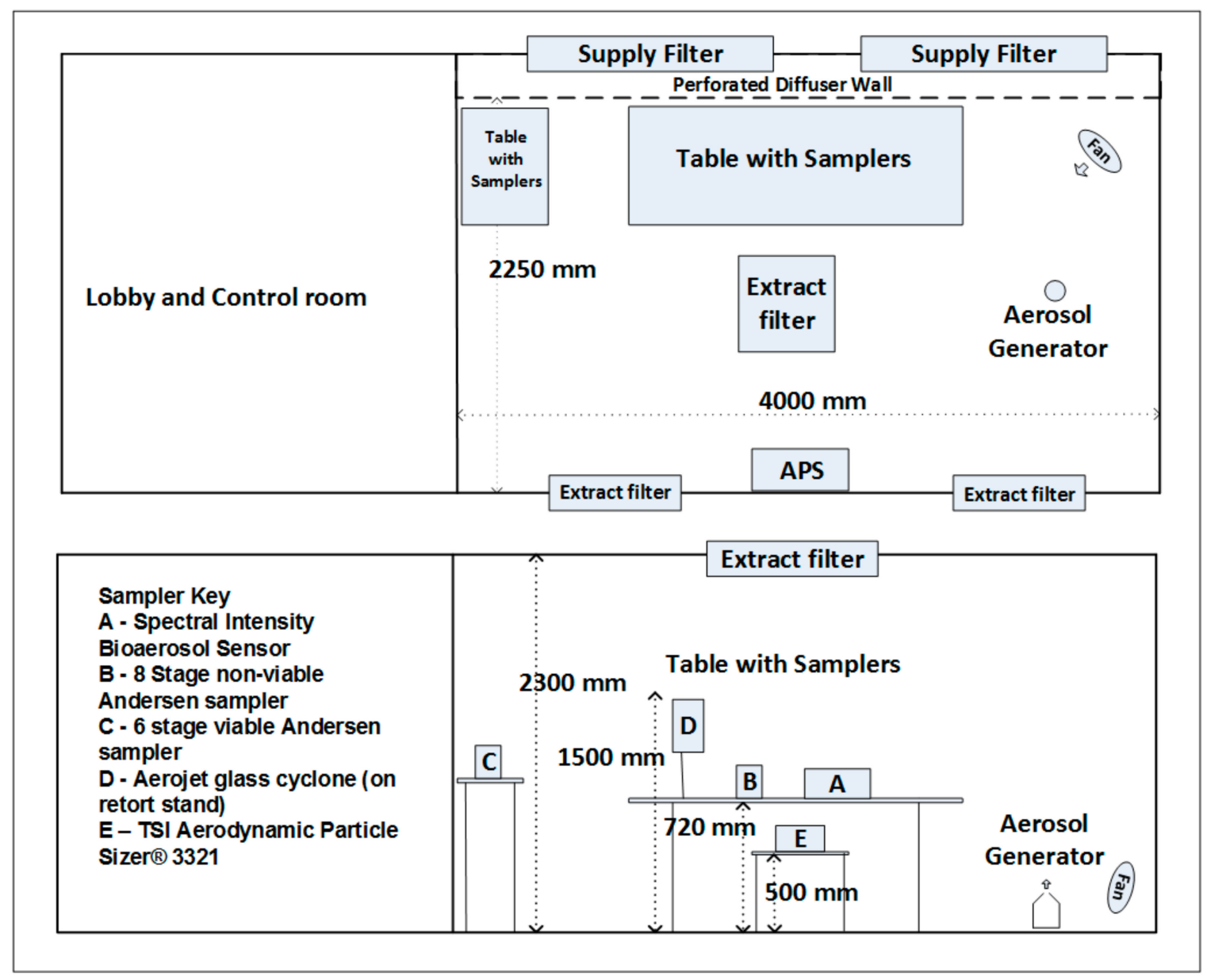

Figure 1. Schematic of test chamber and experimental design.

\subsection{Experimental Design and Instrumentation}

Final grade compost was collected from a green waste compost site (processing organic waste products such as green garden waste, fruit, vegetable waste, straw, stable waste paper and card) and transported to the laboratory in sealed plastic bags and stored at $4{ }^{\circ} \mathrm{C}$. For each experimental run, $25 \mathrm{~g}$ 
of compost was aerosolised using a purpose-built aerosol generator. The purpose was to design and assemble a dry aerosol generation device capable of generating polydisperse aerosol from compost. Figure 2 shows a schematic of the aerosolisation method.

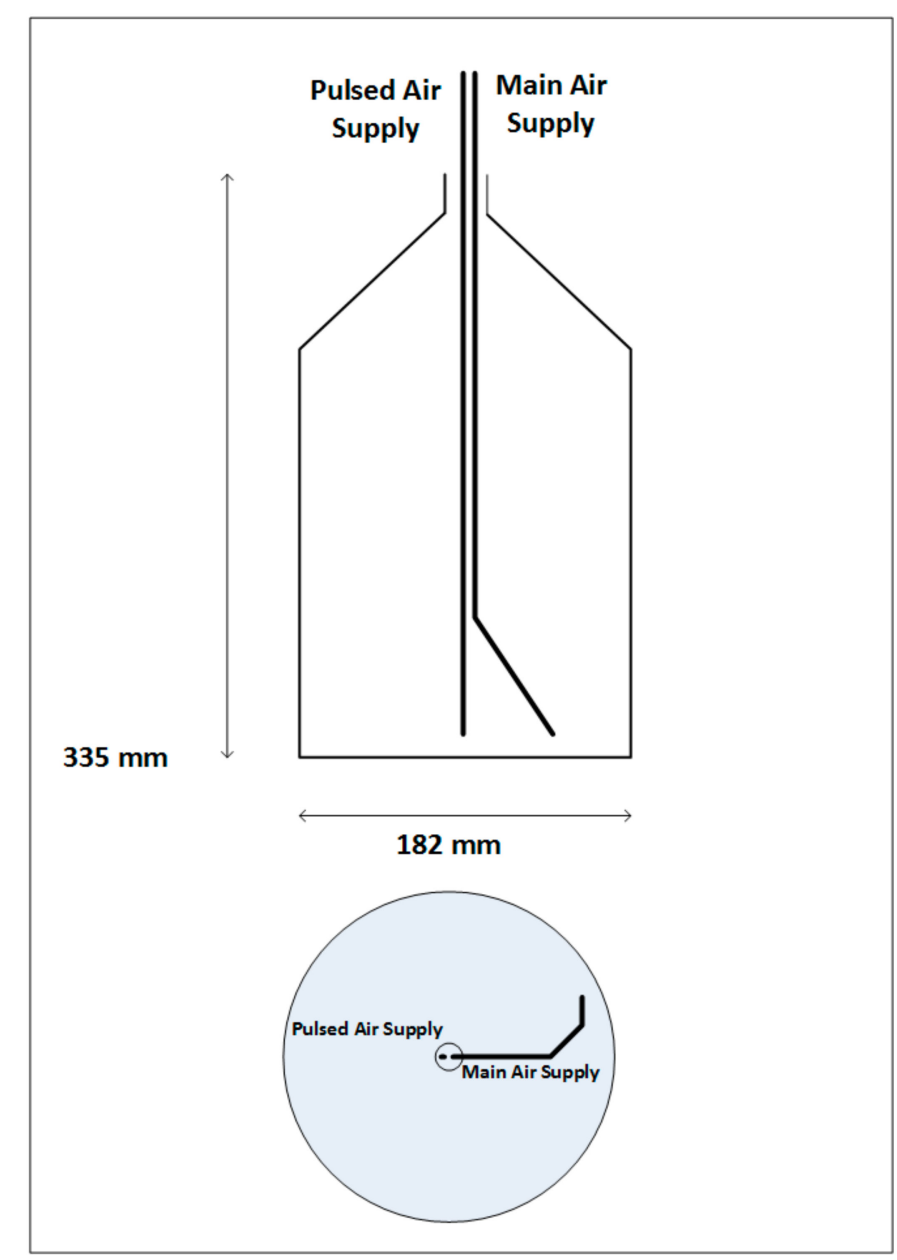

Figure 2. Schematic of aerosol generator.

Compressed air was delivered in to a $5 \mathrm{~L}$ Duran bottle via two lengths of $8 \mathrm{~mm}$ steel pipe introduced into the vessel centrally through the open top. The primary pipe (main air supply) was angled and bent, with a partially closed end, giving a ca. $3 \mathrm{~mm}$ diameter outlet. This delivered air towards the lower edge of the side wall of the bottle, producing a cyclonic effect within the vessel. The second pipe (pulsed air supply) was left straight and positioned with its outlet a few $\mathrm{cm}$ above the centre of the vessel floor. HEPA filtered compressed air was delivered to the vessel via the primary pipe at $20 \mathrm{PSI}$ at a rate of $90 \mathrm{~L} / \mathrm{min}$ and run continuously throughout a sampling period. Due to the nature of the inflowing air, some of the compost collected in the central part of the flask floor. The second pipe (pulsed air supply) was used to deliver short jets of HEPA filtered compressed air to the base of the bottle in order to redistribute the compost as required. The frequency of this operation allowed for increased aerosolisation when required (high aerosolisation). The aerosol concentration inside the chamber was monitored by the direct, live readout of an Aerodynamic Particle Sizer (TSI Incorporated). Several repeat sampling experiments were carried out during periods of no aerosolisation (control) and aerosolisation and each run lasted for $30 \mathrm{~min}$. The aerosolisation runs were performed under two emission scenarios: low and high aerosolisation. A fan was used to aid with the even distribution of aerosols throughout the chamber. A suite of instruments, specific to the detection and quantification of different physico-chemical and biological characteristics of bioaerosol emissions, were deployed inside the chamber at the height of 1-1.5 m (Figure 1 and Table 1). 
Table 1. Instrumentation used in the chamber.

\begin{tabular}{|c|c|c|c|}
\hline Equipment & Analyte & $\begin{array}{c}\text { Sample } \\
\text { Duration (min) }\end{array}$ & $\begin{array}{l}\text { Flow Rate } \\
\text { (L/min) }\end{array}$ \\
\hline Spectral Intensity Bioaerosol Sensor & Total and fluorescent particles & 30 & 0.3 \\
\hline 8 Stage non-viable Andersen Sampler & Endotoxin & 30 & 28.3 \\
\hline 6 stage viable Andersen sampler & $\begin{array}{l}\text { Non-Tuberculous Mycobacteria (NTM) } \\
\text { Legionella }\end{array}$ & $20 \mathrm{~s}$ & 28.3 \\
\hline Aerojet glass cyclone & $\begin{array}{c}\text { Total Viable Counts } \\
\text { Gram negative bacteria } \\
\text { Coliforms } \\
\text { Legionella spp } \\
\text { Non-Tuberculous Mycobacteria (NTM) }\end{array}$ & 5.5 & 600 \\
\hline
\end{tabular}

The chamber was fully ventilated before and after each sampling experiment. The temperature and relative humidity were monitored and were in the ranges of $22-24{ }^{\circ} \mathrm{C}$ and $46-60 \%$, respectively, during various sampling periods.

\subsubsection{Spectral Intensity Bioaerosol Sensor (SIBS)}

The SIBS (Droplet Measurement Technologies, Longmont, CO, USA) is an expansion of the Wideband Integrated Bioaerosol Sensor (WIBS, Droplet Measurement Technologies, Longmont, CO, USA) and differs from the WIBS in recording highly resolved fluorescence intensity measurements across 16 wavelength bands from 298 to $735 \mathrm{~nm}$ for two excitation wavelength ( $\lambda$ ex $285 \mathrm{~nm}$ and $\lambda$ ex $370 \mathrm{~nm}$ ) compared with three emissions ( $\lambda$ em) bands (FL1: $\lambda$ ex $=280 \mathrm{~nm}$, $\lambda \mathrm{em} \sim 310-400 \mathrm{~nm}$, FL2: $\lambda$ ex $=280 \mathrm{~nm}, \lambda \mathrm{em} \sim 420-650 \mathrm{~nm}$, and FL3: $\lambda \mathrm{ex}=370 \mathrm{~nm}, \lambda \mathrm{em} \sim 420-650 \mathrm{~nm}$ ) for the WIBS. Table 2 lists lower and upper wavelength ranges for each channel.

Table 2. Fluorescence measurement channels $(\mathrm{Ch})$ and wavelength ranges.

\begin{tabular}{ccc}
\hline Channel & Lower Wavelength $(\mathbf{n m})$ & Upper Wavelength $(\mathbf{n m})$ \\
\hline 1 & 298.2 & 316.4 \\
2 & 316.4 & 344.8 \\
3 & 344.9 & 362.5 \\
4 & 377.5 & 401.5 \\
5 & 401.5 & 429.7 \\
6 & 430.2 & 457.5 \\
7 & 456.7 & 485.6 \\
8 & 486.0 & 514.0 \\
9 & 514.1 & 542.0 \\
10 & 542.0 & 569.8 \\
11 & 569.9 & 597.6 \\
12 & 597.6 & 625.2 \\
13 & 625.3 & 652.8 \\
14 & 652.8 & 680.2 \\
15 & 680.3 & 707.5 \\
16 & 707.5 & 734.7 \\
\hline
\end{tabular}

The SIBS used in this study derived the equivalent optical diameter (EOD) for particles in the size range $0.4-7 \mu \mathrm{m}$ and had sample flow rate of $0.3 \mathrm{~L} / \mathrm{min}$. Particle size calibration was carried out by Droplet Measurement Technologies, Longmont, CO, USA, using standard monodisperse polystyrene latex microspheres (refractive index: 1.58).

\subsubsection{Aerojet Glass Cyclone}

The glass cyclone sampler is equipped with a liquid injection nozzle at the cyclone entry, allowing for the continuous injection of a collection fluid (Phosphate-buffered mannitol, PBMA), wetting and 
washing the internal surfaces, where captured particles are deposited [17]. The collection fluid was delivered via a peristaltic pump and collected in the base of the sampler during use. It was drawn off using a sterile syringe at the end of the sampling period. Air was drawn through the sampler using a vacuum pump at an approximate sampling rate of $600 \mathrm{~L} / \mathrm{min}$.

\subsubsection{Eight-Stage Non-Viable Andersen Sampler}

The eight-stage non-viable Andersen sampler (Westech, Upper Stondon, UK) is a multi-orifice cascade impactor which allows the collection of aerodynamically size segregated particles at different stages from 0.4 to $>10 \mu \mathrm{m}$. The sampler was loaded with GF/A $81 \mathrm{~mm}$ glass fibre filters (Fisher Scientific, Loughborough, UK) to collect aerosolised compost and operated at $28.3 \mathrm{~L} / \mathrm{min}$ via a calibrated vacuum pump (JS Holdings, Stevenage, UK).

\subsubsection{Six-Stage Viable Andersen Sampler}

The Andersen six-stage viable particle sampler is a multi-orifice cascade impactor offering the collection of aerodynamically sized particles on agar plates [18]. Two samplers were loaded with Legionella and Mycobacteria selective agar plates, Glycine Vancomycin Polymyxin Cycloheximide (GVPC, Oxoid, Basingstoke, UK) and Middlebrooks 7H10 (PHE, Salisbury, UK), respectively, and operated at $28.3 \mathrm{~L} / \mathrm{min}$ via a calibrated vacuum pump (JS Holdings, Stevenage, UK).

\subsection{Sampling and Downstream Bioaerosol Analysis}

Six compost aerosolisation runs of 30 min each were performed under two emission scenarios: low and high. Additionally, control runs (no aerosolisation) were carried out to ensure QA/QC. During the control runs mean number concentration ranged between $0.50-1.14 \mathrm{~cm}^{-3}$ and endotoxin, microbiological culture and qPCR results were negative. Non-tuberculous mycobacteria and Legionella analysis by Anderson six stage viable sampler were conducted for three runs only (one in a low emission scenario and two in high emission scenarios).

\subsubsection{Spectral Intensity Bioaerosol Sensor (SIBS)}

Continuous real-time measurements were made with the SIBS (Droplet Measurement Technologies, Longmont, CO, USA). The SIBS stores single particle data and these were processed in an off line analysis tool kit by choosing an averaging interval of $60 \mathrm{~s}$ and particle size limit of $0.5-7 \mu \mathrm{m}$. Prior to each measurement the SIBS was run in forced trigger mode for five minutes to determine background fluorescence. To set up a fluorescence threshold to calculate fluorescent particle concentration, firstly mean forced trigger values were subtracted from each particle across the channels followed by a single threshold value calculated from mean forced trigger values of all the channels $+3 \times$ mean SD values of all the channels. Furthermore, some particles may not be flashed (excited) during recharge of flash lamps leading to three categories of particles: Total particles, excited particles and fluorescent particles. Therefore, the concentration of fluorescent particles was corrected by Equation (1).

$$
\text { Fluorescent concentration }\left(\mathrm{cm}^{-3}\right)=(\mathrm{F} / \mathrm{E}) \times \mathrm{T}
$$

where $\mathrm{T}$ is total particles $\left(\mathrm{cm}^{-3}\right), \mathrm{E}$ is excited particles $\left(\mathrm{cm}^{-3}\right)$ and $\mathrm{F}$ is fluorescent particles $\left(\mathrm{cm}^{-3}\right)$ (based on the fluorescence threshold).

For the analysis of fluorescent emission intensity across different bands, a five-minute single particle data sample from each aerosolisation run during low and high emission scenario was selected. The sample size for fluorescence emission intensity analysis for different samples is shown in Table 3 . 
Table 3. Sample size of different samples for fluorescence intensity data from the aerosolisation experiments.

\begin{tabular}{cccc}
\hline Emission Scenarios & Sampling 1 & Sampling 2 & Sampling 3 \\
\cline { 2 - 4 } & $\begin{array}{c}\text { Number of } \\
\text { Excited Particles }\end{array}$ & $\begin{array}{c}\text { Number of } \\
\text { Excited Particles }\end{array}$ & $\begin{array}{c}\text { Number of } \\
\text { Excited Particles }\end{array}$ \\
\hline Low emission & 28,004 & 26,782 & 25,297 \\
High emission & 22,096 & 30,374 & 31,633 \\
\hline
\end{tabular}

For each sample, firstly, mean forced intensity values were subtracted from individual particles in corresponding channels and the particles with emission intensity values less than forced intensity values in all channels were excluded. Then, mean fluorescence intensity of fluorescent particles was computed across the emission wavelength bands on a channel-by-channel basis. Thus, three fluorescence spectra were obtained for each scenario. Lastly, mean emission intensities along with standard deviation were computed for low and high emission scenarios.

\subsubsection{Endotoxin}

Before sampling filters, tweezers and glassware were depyrogenated by heating to $250{ }^{\circ} \mathrm{C}$ for at least $4 \mathrm{~h}$. After sampling, each filter was removed and stored in $15 \mathrm{~mL}$ of LAL water for single filters or $40 \mathrm{~mL}$ of LAL water for multiple filters. In total nine size fractions were obtained with some filters extracted together to reduce the fractionation profile to $10.0-9.0 \mu \mathrm{m}, 9.0-2.1 \mu \mathrm{m}$, 2.1-0.43 $\mu \mathrm{m}$ and $<0.43 \mu \mathrm{m}$. To extract the endotoxin, filters were shaken for $1 \mathrm{~h}$ at room temperature. The supernatant was transferred to a clean tube and centrifuged at $1000 \times g$ for $15 \mathrm{~min}$ to remove any glass fibre. The clear supernatant was assayed for endotoxin using a kinetic chromogenic Limulus Amoebocyte Lysate (LAL) assay kit (Associates of Cape Cod, Inc., East Falmouth, MA, USA). Briefly, $50 \mu \mathrm{L}$ of sample was added to a 96 well microplate with $50 \mu \mathrm{L}$ of lysate reconstituted with Glucashield to prevent glucan interference in the test. A standard curve was obtained using control standard endotoxin ranging 5-0.005 EU/mL. Endotoxin concentrations were calculated by incubating the plate at $37{ }^{\circ} \mathrm{C}$ in a microplate reader with a $405 \mathrm{~nm}$ filter for $90 \mathrm{~min}$. Each sample was assayed in duplicate.

\subsubsection{Microbial Culture and Identification}

\section{Raw Compost}

To characterise the microbial composition of the raw compost material, five grams of compost was re-suspended in $30 \mathrm{~mL}$ sterile distilled water by shaking at $100 \mathrm{rpm}$ for $10 \mathrm{~min}$. After $15 \mathrm{~min}$ of settling $100 \mu \mathrm{L}$ aliquots (and 10-fold serial dilutions) in duplicate were plated on Legionella selective GVPC (Glycine, Vancomycin hydrochloride, Polymyxin B sulphate, Cycloheximide; ThermoFisher, Loughborough, UK) following acid and heat treatment according to ISO 11731 [19]. Plates were incubated at $30^{\circ} \mathrm{C} \pm 1{ }^{\circ} \mathrm{C}$ and $37^{\circ} \mathrm{C} \pm 1{ }^{\circ} \mathrm{C}$ for 10 days. To enumerate total viable count (TVC), Gram negative organisms, total coliforms and E. coli, $100 \mu \mathrm{L}$ (and serial dilutions) were plated on Trypticase Soya agar (TSA), MacConkey and Brilliance ${ }^{\mathrm{TM}}$ E. coli/coliform Selective Agar (COLIC; ThermoFisher, Loughborough, UK). Plates were incubated at $37^{\circ} \mathrm{C} \pm 1{ }^{\circ} \mathrm{C}$ for three days. For the isolation of Mycobacteria, $100 \mu \mathrm{L}$ of sample was decontaminated using 2\% $\mathrm{NaOH}$ (Mycoprep, BD, Franklin Lakes, NJ, USA) for 15 min prior to plating on Middlebrooks 7H11 agar and incubating for up to 42 days in duplicate at $30^{\circ} \mathrm{C} \pm 1{ }^{\circ} \mathrm{C}$ and $37^{\circ} \mathrm{C} \pm 1{ }^{\circ} \mathrm{C}$.

\section{Microbiological Analysis of Air Samples by Culture and MALDI-TOF}

Legionella and Mycobacteria selective agar plates from the six-stage viable Andersen samplers were incubated at $37{ }^{\circ} \mathrm{C} \pm 1{ }^{\circ} \mathrm{C}$ for 10 and 42 days, respectively. Liquid concentrates $(100 \mu \mathrm{L})$ from the cyclone samplers were cultured for Legionella, Mycobacteria, TVC, Gram negative bacteria 
and total coliforms/E. coli as described for raw compost samples. Representative examples of all colony types on TSA, MacConkey and COLIC agar as well as colonies with morphologies consistent with Legionella and Mycobacteria were identified by Matrix Assisted Laser Desorption and Ionisation Time of Flight Mass Spectrometry (MALDI-TOF) using the Bruker Biotyper (Bruker, Coventry, UK). Presumptive Mycobacteria isolates were subject to a full bead-extraction protocol prior to MALDI-TOF according to the manufacturer's instructions [20]. The degree of similarity between an unknown isolate and a reference sample is determined by the MALDI Biotyper 2.0 software by comparing peak profiles and calculating a logarithmic score between 0 and 3 . These log scores were used as cut-offs for bacterial identification. Log scores, as recommended by the manufacturer, were used as cut-offs for bacterial identification. Scores $\geq 2.000$ indicated reliable species-level identification, while scores between 1.700 and 1.999 indicated genus-level identification.

Quantitative Polymerase Chain Reaction

Due to the expected abundance of microorganisms in compost and previous inhibition of Legionella culture [21] qPCR was employed to facilitate detection of Legionella from raw compost and air samples. For cyclone concentrates, a minimum of $1 \mathrm{~mL}$ was concentrated by membrane filtration using $0.2 \mu \mathrm{m}$ polycarbonate filters (Pall, Portsmouth, UK) prior to DNA extraction using the DNeasy PowerWater DNA isolation kit (Qiagen, Manchester, UK) according to the manufacturer's instructions. For raw compost samples, DNA was extracted from triplicate samples of $0.25 \mathrm{~g}$ using the DNeasy PowerSoil DNA isolation kit (Qiagen) according to the manufacturer's instructions. DNA was eluted in a final volume of $100 \mu \mathrm{L}$. Quantitative PCR (qPCR) was performed for Legionella spp., Legionella pneumophila and L. pneumophila sg-1 as previously described [22] with the following modifications: TaqMan environmental mastermix beads incorporating a $3^{\prime}$ VIC labelled internal control (Thermofisher) to monitor for inhibition were used for all reactions. The final reaction volume was $30 \mu \mathrm{L}$. Reaction conditions were $95^{\circ} \mathrm{C}$ for $15 \mathrm{~min}$ followed by 40 cycles of $95^{\circ} \mathrm{C}$ for $10 \mathrm{~s}$ and $60^{\circ} \mathrm{C}$ for $1 \mathrm{~min}$. Legionella lognbeachae qPCR was conducted using the Genesig ${ }^{\circledR}$ Legionella longbeachae standard kit (Primerdesign, Southampton, UK) according to the manufacturer's instructions. All reactions were performed in an ABI 7500 FAST thermocycler (Thermofisher, UK). Filter blanks, no template controls (PCR grade water) and positive controls (either L. pneumophila sg-1 NCTC 12821 or L. longbeachae NCTC 11477) were run with each set of samples. Data were analysed using the ABI 7500 v2.3 Software. The limits of detection and quantification were $4.0 \times 10^{2}$ genome units (GU)/g and $8.0 \times 10^{2} \mathrm{GU} / \mathrm{g}$, respectively, for raw compost and $4.6 \times 10^{1}(\mathrm{GU}) / \mathrm{m}^{3}$ and $9.2 \times 10^{1} \mathrm{GU} / \mathrm{m}^{3}$, respectively, for air samples.

\section{Results and Discussion}

\subsection{SIBS}

\subsubsection{Number Concentration}

The concentration of total and fluorescent particles was highest during the high emission scenario although the number weighted fractions of fluorescent particles were comparable between the two emission scenarios (Table 4).

Table 4. Summary of particle concentrations measured by the SIBS (n, number of measurements; NT, Number of total Particles; NF, Number of fluorescent particles; SD, Standard deviation).

\begin{tabular}{ccccc}
\hline & \multicolumn{2}{c}{ Low Emission $(\boldsymbol{n}=\mathbf{3})$} & \multicolumn{2}{c}{ High Emission $(\boldsymbol{n}=\mathbf{3})$} \\
\cline { 2 - 5 } & Mean & SD & Mean & SD \\
\hline $\mathrm{NT}\left(\mathrm{cm}^{-3}\right)$ & 51 & 15 & 129 & 25 \\
$\mathrm{NF}\left(\mathrm{cm}^{-3}\right)$ & 11 & 3 & 33 & 8 \\
$\mathrm{NF} / \mathrm{NT}$ & 0.22 & 0.01 & 0.26 & 0.02 \\
\hline
\end{tabular}




\subsubsection{Fluorescence Spectra}

Figure 3 presents fluorescence intensity spectra for two excitation wavelengths under low and high emission scenarios. The samples from low emission scenarios showed four fluorescence modes for excitation wavelength ( $\lambda$ ex) $285 \mathrm{~nm}$ in increasing order of emission wavelengths ( $\lambda \mathrm{em}$ ) $456.7-485.6 \mathrm{~nm}$ (Ch 7), 542-569.8 nm (Ch 10), 597.6-625.2 nm (Ch 12) and 652.8-680.2 nm (Ch 14), while, for excitation

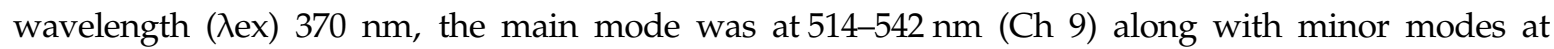
597.6-625.2 nm (Ch 12) and 652.8-680.2 nm (Ch 14). In contrast, during high emission scenarios the dominant peaks for both $\lambda$ ex 285 and $370 \mathrm{~nm}$ were comparable to low emission scenarios but slights shifts were observed in secondary modes (Figure 3). For example, during high emission scenarios, there was a distinct shift from $298.2-316.4 \mathrm{~nm}$ (Ch 1) to 316.4-344.8 nm (Ch 2), 542-569.8 nm (Ch 10) to 569.9-597.6 nm (Ch 11) for $\lambda$ ex $285 \mathrm{~nm}$ and 597.6-625.2 nm (Ch 12) to 569.9-597.6 nm (Ch 11) and 652.8-680.3 nm (Ch 14) to $680.3-707.5 \mathrm{~nm}$ (Ch 15) for $\lambda$ ex $370 \mathrm{~nm}$ in comparison to low emission scenarios.

The potential presence of a host of bio-fluorophores in the compost sample and their overlapping $\lambda \mathrm{ex} / \lambda \mathrm{em}$ hinder the exact assignment of fluorescence to a specific bio-fluorophore. Nevertheless, the existing information on excitation and emission spectra of several types of fluorescent molecules [23-26] can help elucidate the molecular composition of distinct spectral features from biological materials. Different fluorescence peaks during low and high emissions scenarios of aerosolized compost could be assigned to amino acids, structural compounds, coenzymes, pigments and secondary metabolites, for instance amino acids in Ch 1-3; cellulose, chitin, lignin and pyridoxamine in Ch 4 , Ch 5 and Ch7; flavins in Ch 9 and Ch10; age-related pigments in Ch 11; and terpenoids in Ch 14 for $\lambda$ ex 285. The similar assignment could be informed for different fluorescence peaks for $\lambda$ ex 370 . Furthermore, improved spectral resolution by SIBS reveals additional information in comparison to the WIBS with the three emission bands FL1 ( $\lambda$ ex $=280 \mathrm{~nm}, \lambda \mathrm{em} \sim 310-400 \mathrm{~nm})$, FL2 $(\lambda \mathrm{ex}=280 \mathrm{~nm}, \lambda \mathrm{em} \sim 420-650 \mathrm{~nm})$ and FL3 ( $\lambda$ ex $=370 \mathrm{~nm}, \lambda \mathrm{em} \sim 420-650 \mathrm{~nm}$ ) (Figure 3).

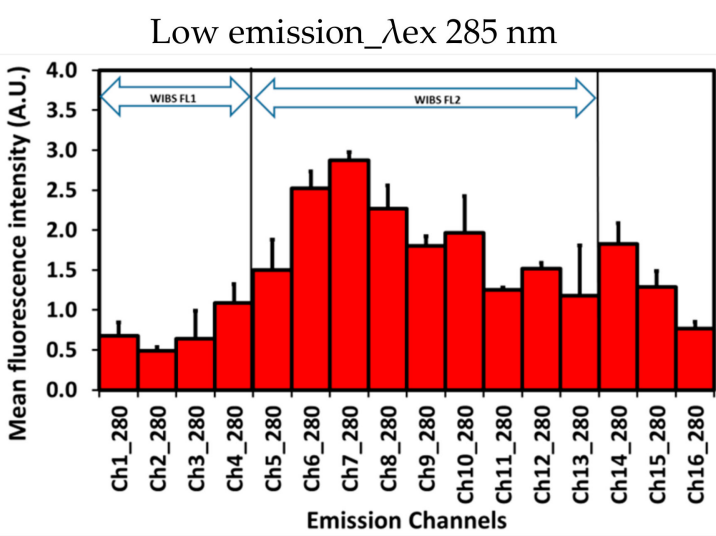

High emission_ $\lambda$ ex $285 \mathrm{~nm}$

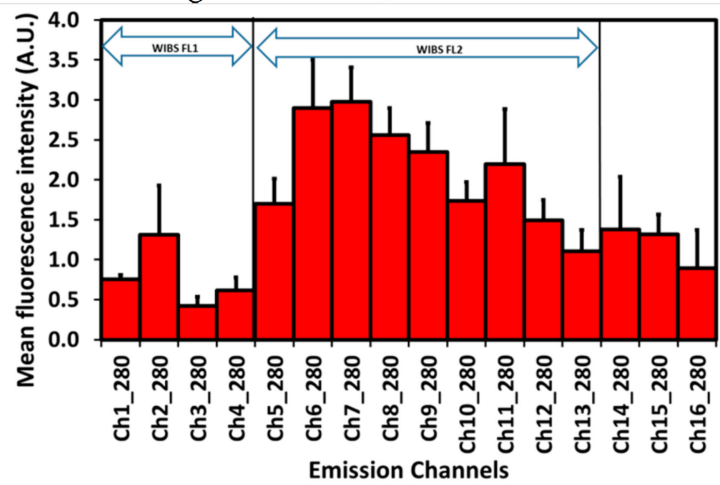

Low emission_lex $370 \mathrm{~nm}$

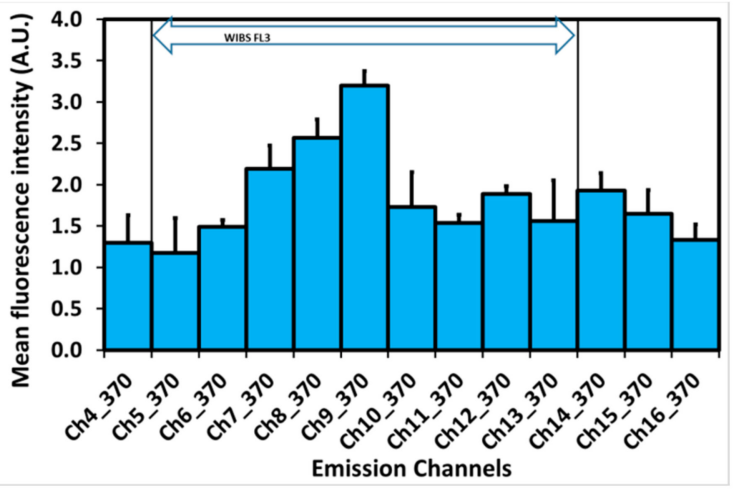

High emission_tex $370 \mathrm{~nm}$

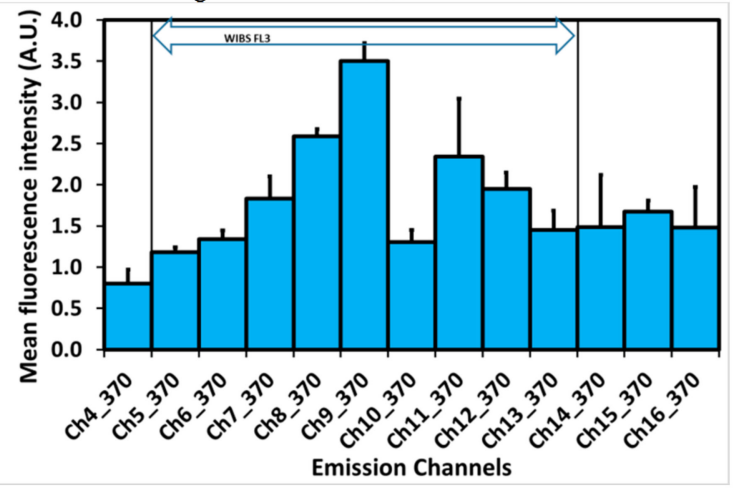

Figure 3. Representative fluorescence intensity across different emission channels for two excitation wavelengths during low and high emission scenarios. Bars = standard deviation. 
These spectra with multiple fluorescence peaks can help to elucidate spectrally integrated signals of biological materials and improve selectivity in LIF based detection systems. For example, improved selectivity in fluorophores assignment to peak emissions in a spectrum can advance discrimination algorithms by using ratios between different fluorescence modes. However, there is a need to improve particle classification certainty and to develop numerical methods to exploit emission spectra data to enhance the selectivity of LIF based detection systems. The particle classification and data analysis methodologies proposed and used for WIBS data $[27,28]$ can be extended to SIBS data but are yet to be tested. With reference to the size of particles in the samples analysed for fluorescence spectra, the majority of particles were in the fine size fraction (Figure 4). A slight increase in mean particle size during high emission $(1.31 \pm 1.23 \mu \mathrm{m})$ in comparison to low emission $(1.27 \pm 1.21 \mu \mathrm{m})$ was observed. A considerable fraction of fluorescent particles was submicron (below $1 \mu \mathrm{m}$ ) and fluorescence in these particles from compost aerosolisation under controlled conditions raises questions, such as: What is the molecular origin of observed fluorescence in submicron particle size range? This may reflect the fluorescing contribution from cellular fragments or molecular decomposition products of biological material in the compost or instrument artifact. Viruses and protein toxins have been reported to have one or more fluorescing amino acids [29]. Similarly, humic-like substances have also been reported as potential interfering non-biological compounds exhibiting a range of spectral modes [24]. However, the exact origin of fluorescence in submicron particles needs further investigation.

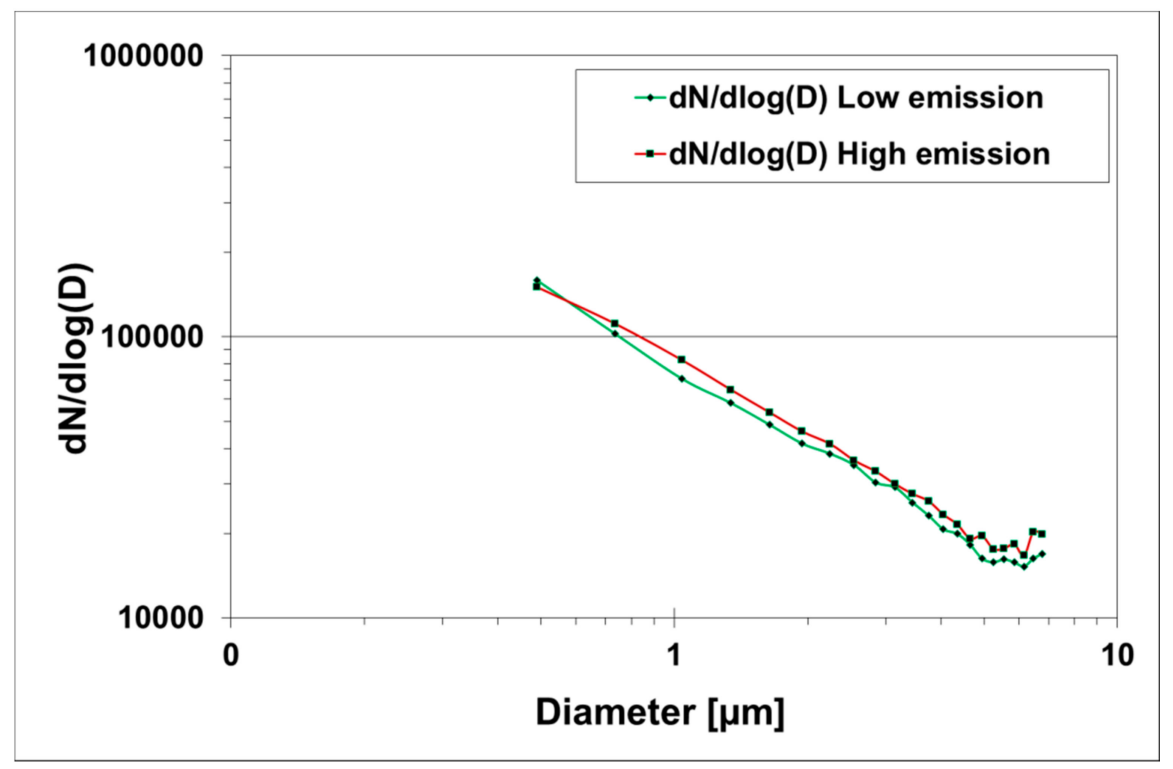

Figure 4. Size distribution of fluorescent particles in the samples analysed for fluorescence spectra under low and high emission scenarios.

\subsection{Endotoxin}

Total endotoxin concentrations averaged $253 \mathrm{EU} \mathrm{m}^{-3}( \pm 72)$ and $1245 \mathrm{EU} \mathrm{m}^{-3}( \pm 127)$ under low and high emission scenarios, respectively. Size fractionation showed endotoxin concentrations were highest in the 2.1-9 $\mu \mathrm{m}$ size range for both scenarios (58\% and $65 \%$ for low and high scenarios) (Figure 5A). However, a considerable amount was also associated with $0.43-2.1 \mu \mathrm{m}$ (27\% and $15 \%$ for low and high scenarios). Further analysis of size distribution showed that mass median aerodynamic diameter (MMAD) shifted from $2.44 \mu \mathrm{m}$ (GSD 2.14) during low emission to $4.11 \mu \mathrm{m}$ (GSD 1.84) in high emission scenarios (Figure $5 \mathrm{~B}$ ). This shows that the distribution of endotoxin was shifted towards higher size particles during high emission scenarios probably due to an increase in coarse size particles aerosolised. 
(A)

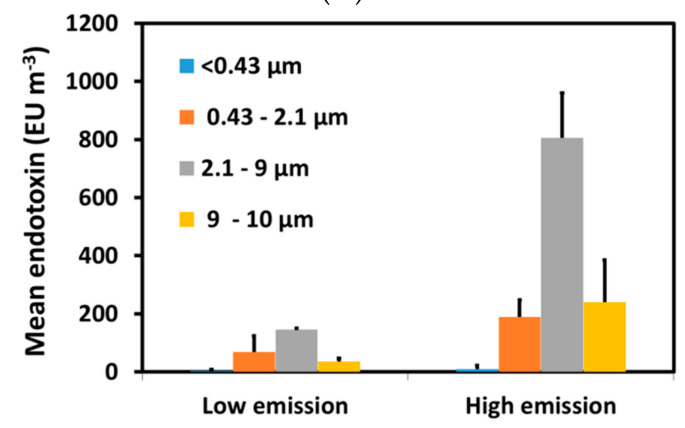

(B)

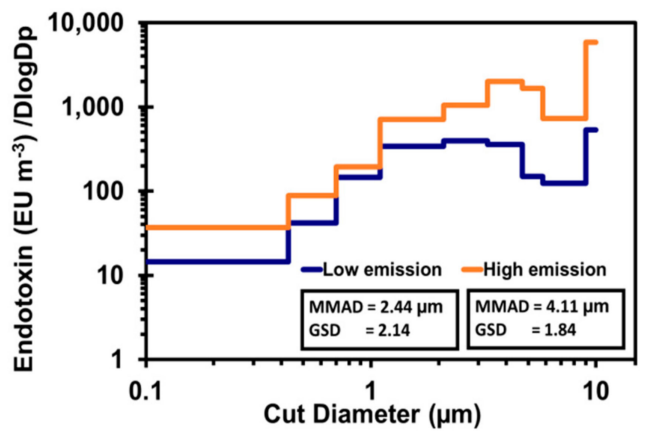

Figure 5. (A) Size fractionated endotoxin concentration under different emission scenarios ( $\mathrm{n}=3$ measurements each; Bars = Standard deviation); and (B) size distribution of endotoxin during low and high emission ( $\mathrm{n}=1$ measurement each).

Endotoxin is a significant component of bioaerosols from composting facilities as it is known to trigger an immune response in humans [30]. Limited work has been undertaken on the size fractionation of endotoxin and to our knowledge none under controlled conditions. Previous studies have shown that endotoxin is associated with the coarse particle fraction [31], to some extent, this is likely due to larger particles being able to retain a larger amount of endotoxin. However, size distribution analysis has shown that a significant concentration of endotoxin is associated with the fine fraction $(<2.1 \mu \mathrm{m})$ as well which could have implications for how far it may be able to travel in the environment and how deep into the lung it can penetrate.

\subsection{Microbial Culture and $q P C R$}

A range of microorganisms were identified in this study at high concentrations in the raw compost and under both low and high emission scenarios (Table 5). High concentrations of microorganisms, including Gram negatives, were detected in the air for both low and high emission runs $\left(1.91 \times 10^{5} \mathrm{CFU} \mathrm{m}^{-3}\right.$ and $3.84 \times 10^{5}$ total viable counts and $1.88 \times 10^{4} \mathrm{CFU} \mathrm{m}^{-3}$ and $1.79 \times 10^{5} \mathrm{Gram}$ negative bacteria, respectively). The most commonly isolated organism $\left(10^{4} \mathrm{CFUm}^{-3}\right)$ as identified by MALDI-TOF was Staphylococcus sciuri (Table 6). This is a reflection of the raw material where $S$. sciuri was also the predominant organism $\left(6.0 \times 10^{7} \mathrm{CFU} \mathrm{g}{ }^{-1}\right)$. Other organisms detected in the air at significant concentrations include Acinetobacter spp. $\left(10^{2} \mathrm{CFU} \mathrm{m}{ }^{-3}\right)$ and Bacillus spp. $\left(10^{3} \mathrm{CFU} \mathrm{m}{ }^{-3}\right)$ (Table 6). High concentrations of coliforms were detected in raw compost $\left(1.68 \times 10^{7} \mathrm{CFU} \mathrm{g}^{-1}\right)$ and air during low $\left(2.27 \times 10^{2} \mathrm{CFU} \mathrm{m}{ }^{-3}\right)$ and high $\left(2.59 \times 10^{3} \mathrm{CFU} \mathrm{m}^{-3}\right)$ emission conditions (Table 5). In the air samples, the following coliforms were detected: Enterobacter cloacae, Enterobacter aerogenes and Enterococcus casseliflavus (Table 6). All are clinically significant human pathogens and indicate potential contamination of the compost with faecal material.

All cyclone samples (including those that were pre-treated with NaOH-NALC as per standard decontamination protocols) were overgrown with fungi within $72 \mathrm{~h}$ precluding the growth and isolation of NTM. However, NTM were detected in both raw compost and Andersen air samples total concentrations between $9.5 \times 10^{2} \mathrm{CFU} \mathrm{m}{ }^{-3}$ and $2.5 \times 10^{4} \mathrm{CFU} \mathrm{m}{ }^{-3}$ (mean $1.3 \times 10^{4} \mathrm{CFU} \mathrm{m}{ }^{-3}$ ). Mycobacterium smegmatis, M. hassiacum and M. phlei (maximum concentrations of $8.5 \times 10^{2} \mathrm{CFU} \mathrm{m} \mathrm{m}^{3}, 1.7 \times 10^{3} \mathrm{CFU} \mathrm{m}^{-3}$, and $2.1 \times 10^{2} \mathrm{CFU} \mathrm{m} \mathrm{m}^{-3}$ respectively) were identified by MALDI-TOF. None are of significant public health concern and not generally considered pathogenic to humans. NTM predominated in the 3.3-4.7 $\mu \mathrm{m}$ fraction of the Andersen sampler with $41 \%$ of the total CFU. However, $40 \%$ of the total CFU was associated with $3.3-0.65 \mu \mathrm{m}$ fraction and the count median aerodynamic diameter was $1.67 \mu \mathrm{m}$ which has the potential to be respirable in the lung. The results demonstrate that significant concentrations of respirable NTM can be aerosolised and this may pose a public health risk should NTM organisms of concern be present. However, the true public health significance of this is unknown and remains to be elucidated. 
Table 5. Summary of microbiological findings.

\begin{tabular}{cccccc}
\hline & Raw Compost & \multicolumn{2}{c}{$\begin{array}{c}\text { Low Emission } \\
(\boldsymbol{n}=\mathbf{3})\end{array}$} & \multicolumn{2}{c}{$\begin{array}{c}\text { High Emission } \\
(\boldsymbol{n}=\mathbf{3})\end{array}$} \\
\cline { 2 - 7 } & $\begin{array}{c}\text { Mean } \\
\left(\mathbf{g}^{-1} \mathbf{)}\right.\end{array}$ & $\begin{array}{c}\text { Mean } \\
\left(\mathbf{m}^{-3}\right)\end{array}$ & SD & $\begin{array}{c}\text { Mean } \\
\left(\mathbf{m}^{-3}\right)\end{array}$ & SD \\
\hline Total viable count (CFU) & $9.66 \times 10^{8}$ & $1.91 \times 10^{5}$ & $5.10 \times 10^{4}$ & $3.84 \times 10^{5}$ & $2.11 \times 10^{5}$ \\
Gram negative bacteria (CFU) & $6.00 \times 10^{7}$ & $1.88 \times 10^{4}$ & $3.06 \times 10^{4}$ & $1.79 \times 10^{5}$ & $2.42 \times 10^{5}$ \\
Coliforms (CFU) & $1.68 \times 10^{7}$ & $2.27 \times 10^{2}$ & $1.52 \times 10^{2}$ & $2.59 \times 10^{3}$ & $1.93 \times 10^{3}$ \\
Legionella spp. (GU) & $3.28 \times 10^{4}$ & $1.26 \times 10^{3}$ & $6.50 \times 10^{2}$ & $9.55 \times 10^{2}$ & $2.92 \times 10^{2}$ \\
L. pneumophila (GU) & $2.93 \times 10^{4}$ & $1.34 \times 10^{3}$ & $7.76 \times 10^{2}$ & $5.43 \times 10^{2}$ & $1.66 \times 10^{2}$ \\
L. pneumophila sg-1 (GU) & $1.46 \times 10^{4}$ & $1.36 \times 10^{3}$ & $1.00 \times 10^{3}$ & $2.44 \times 10^{2}$ & $4.36 \times 10^{1}$ \\
\hline
\end{tabular}

Legionella was not detected by culture using either cyclone or Andersen sampling probably due to gross contamination of the selective media with other non-Legionella organisms. Legionella species were detected in the cyclone concentrates using $\mathrm{qPCR}$ at concentrations between $6.91 \times 10^{2}$ and $1.11 \times 10^{3} \mathrm{GU} \mathrm{m}^{-3}$ (mean $1.26 \times 10^{3} \mathrm{GU} \mathrm{m}^{-3}$ ) during low emission scenarios and from $6.21 \times 10^{2}$ to $1.17 \times 10^{3}$ (mean $9.55 \times 10^{2}$ ) in high emissions scenarios. The mean concentration of Legionella pneumophila was $1.34 \times 10^{3}$ and $5.43 \times 10^{2} \mathrm{GU} \mathrm{m}^{-3}$ for low and high emissions. While the mean concentration of L. pneumophila serogroup (sg) 1 (the causative agent of $>80 \%$ of Legionnaires' disease cases [32] was $1.36 \times 10^{4}$ and $2.4 \times 10^{2} \mathrm{GU} \mathrm{m}^{-3}$ for low and high emission scenarios. Importantly, the data show that the majority of the Legionella spp. in the air was Legionella pneumophila and the majority of the L. pneumophila was L. pneumophila sg-1. This is a reflection of the concentrations detected in the raw compost (Table 5). It is unclear why the Legionella recoveries were generally higher in the low emission scenario. It may be partially due to Legionella being more associated with the lower size fractions of the aerosol which were more common in the low emissions scenario. Whilst Legionella has previously been detected in compost samples [33], to our knowledge, this is the first time that aerosolisation of Legionella pneumophila from green waste compost has been demonstrated. Legionella longbeachae, the agent commonly associated with Legionnaires' disease, from compost/soil was not detected in either the raw compost or the air by culture or qPCR. This is surprising as previous studies have shown a high prevalence of L. longbeachae in compost.

Table 6 lists the representative organisms in aerosolised air samples identified by MALDI-TOF. All organisms were also detected in the raw compost. Staphylococcus sciuri dominated in both the raw material and the air samples. $\mathrm{CFU} \mathrm{\textrm {m } ^ { - 3 }}$ values are given as approximations only as not every colony was confirmed by MALDI-TOF. During low emission scenarios, one of the samples had the predominance of Bacillus species that was not seen in other samples. Escherichia coli which has potential public health implications and is a marker of faecal contamination was detected in the raw material at a concentration of $3.42 \times 10^{4} \mathrm{CFU} \mathrm{g}^{-1}$. However, it was only detected in one air sample suggesting it does not readily survive aerosolisation under these controlled conditions.

Table 6. Predominant organisms detected by MALDI-TOF in air samples during low and high emission scenarios (approximate concentration $\mathrm{CFU} \mathrm{m}{ }^{-3}$ ).

\begin{tabular}{cccc}
\hline High Emission & \multicolumn{2}{c}{ Low Emission } \\
\hline Microbial sp. & CFU m ${ }^{-3}$ & Microbial sp. & CFU m $^{-3}$ \\
\hline Staphylococcus sciuri & $10^{4}$ & Staphylococcus sciuri & $10^{4}$ \\
Serratia rubidaea & $10^{3}$ & Bacillus firmus & $10^{3}$ \\
Sphingobacterium mizutaii & $10^{3}$ & Bacillus pumilus & $10^{3}$ \\
Bordetella petrii & $10^{3}$ & Bacillis clausii & $10^{3}$ \\
Nocardia paucivorans & $10^{2}$ & Bacillus niacin & $10^{3}$ \\
Escherichia coli & $10^{2}$ & Enterobacter cloacae & $10^{2}$ \\
Acinetobacter spp. & $10^{2}$ & Nocardia paucivorans & $10^{2}$ \\
Enterobacter cloacae & $10^{2}$ & Bordetella petrii & $10^{2}$ \\
Staphylococcus hominis & $10^{2}$ & Sphingobacterium mizutaii & $10^{2}$ \\
Paenicacillus residui & $10^{2}$ & Brevundimonas diminuta & $10^{2}$ \\
Enterobacter casseliflavus & $10^{2}$ & Ochrobactrum intermedium & $10^{2}$ \\
Enterobacter aerogenes & $10^{2}$ & & \\
Pseudomonas putida & $10^{2}$ & & \\
\hline
\end{tabular}




\section{Conclusions}

Aerosolised green waste compost, as test material, was comprehensively analysed using different traditional and enhanced methods for a range of bioaerosols and their components with the aim of advancing bioaerosol hazard characterisation of complex industrial sources. A real time bioaerosol sensor (Spectral Intensity Bioaerosol Sensor) was deployed for the first time in combination with other samplers. The emission spectra were multimodal and a slight shift in emission wavelengths was observed in secondary modes during high emission scenario. It has demonstrated the potential to provide rapid, instantaneous, total and fluorescent particle monitoring which could help to streamline future monitoring regimes. However, improved data analysis techniques as well as laboratory studies with relevant biological fluorophore/materials are required for the elucidation of spectrally integrated signals and thus improving emission characterisation. Real time monitoring of bioaerosol could inform development and validation of proxies for bioaerosol emission such as particulate monitoring with low cost sensors. Size fractionation of endotoxin-associated particles showed that, whilst a dominant proportion of endotoxin was associated with coarse size fractions, $17-27 \%$ was found in fine size fraction. Significant concentrations of microorganisms were detected in both raw compost and air samples including coliforms and Legionella species. Aerosolisation of Legionella pneumophila from green waste compost has been demonstrated for the first time. The study provides information on comparisons of physico-chemical and biological characteristics of bioaerosols emissions measured by diverse means and provide baseline training data with respect to various enhanced methods/techniques to characterise bioaerosol emissions from industrial sources. It will therefore inform the design of monitoring approaches to be used under real-world scenarios.

This study has limitations. Only one compost sample was examined as a complex source material and the data cannot be taken as representative of all compost-derived bioaerosols. Future researchers should avoid using our data in an indiscriminate way as source-term data for models of composting emission and dispersion. A discrete but suitable range of microbiological analysis was conducted. There are other organisms of public health significance that could vary with the relevant emission sources. Finally, qPCR does not differentiate between viable and non-viable cells and, in the absence of successful culture evidence, careful interpretation of the Legionella data is therefore required. Next generation sequencing could be used in the future to study the microbial composition at higher resolution.

Author Contributions: Conceptualization, Z.A.N., C.R., T.L.G., E.H., B.W., S.K., S.J., A.B. and S.T.; Data curation, Z.A.N., C.R., S.C. and D.S.; Formal analysis, Z.A.N.; Funding acquisition, T.G., E.H., S.J., A.B. and S.T.; Investigation, Z.A.N., C.R., S.C., S.K. and S.P.; Methodology, Z.A.N., C.R., S.C. and S.P.; Project administration, S.T.; Resources, T.L.G., S.J., A.B. and S.T.; Supervision, S.T.; Visualization, Z.A.N. and C.R.; Writing-original draft, Z.A.N., C.R. and S.C.; and Writing-review and editing, Z.A.N., S.C., T.L.G., E.H., B.W., S.J., A.B., R.P.K., K.W., S.J.T.P., G.D., S.G.A., F.C. and S.T.

Funding: This research was funded by the Natural Environment Research Council (NE/M01163/1, NE/M011747/1, NE/M011763/1, NE/M011658/1 and NE/M010961/10). The APC was funded by (NE/M01163/1).

Acknowledgments: This award is made under the auspices of the Environmental Microbiology and Human Health programme. This work represents the views of the authors, and not necessarily those of the funders and authors' associated institutions or organisations. The data will be available on 1 July 2020 via the Environmental Information Data Centre, the Natural Environment Research Council's long-term data repository for terrestrial and freshwater sciences https:// catalogue.ceh.ac.uk/eidc/documents.

Conflicts of Interest: The authors declare no conflicts of interest. 


\section{References}

1. Douglas, P.; Bakolis, I.; Fecht, D.; Pearson, C.; Sanchez, M.L.; Kinnersley, R.; de Hoogh, K.; Hansell, A.L. Respiratory hospital admission risk near large composting facilities. Int. J. Hyg. Environ. Health 2016, 219, 372-379. [CrossRef] [PubMed]

2. Borlee, F.; Yzermans, C.J.; van Dijk, C.E.; Heederik, D.; Smit, L.A.M. Increased respiratory symptoms in COPD patients living in the vicinity of livestock farms. Eur. Respir. J. 2015, 46, 1605-1614. [CrossRef] [PubMed]

3. Pearson, C.; Littlewood, E.; Douglas, P.; Robertson, S.; Gant, T.W.; Hansell, A.L. Exposures and Health Outcomes in Relation to Bioaerosol Emissions From Composting Facilities: A Systematic Review of Occupational and Community Studies. J. Toxicol. Environ. Health Part B 2015, 18, 43-69. [CrossRef] [PubMed]

4. $\quad$ Douwes, J.; Thorne, P.; Pearce, N.; Heederik, D. Bioaerosol health effects and exposure assessment: Progress and prospects. Ann. Occup. Hyg. 2003, 47, 187-200. [PubMed]

5. Persoons, R.; Parat, S.; Stoklov, M.; Perdrix, A.; Maitre, A. Critical working tasks and determinants of exposure to bioaerosols and MVOC at composting facilities. Int. J. Hyg. Environ. Health 2010, 213, 338-347. [CrossRef] [PubMed]

6. Fathi, S.; Hajizadeh, Y.; Nikaeen, M.; Gorbani, M. Assessment of microbial aerosol emissions in an urban wastewater treatment plant operated with activated sludge process. Aerobiologia 2017, 33, 507-515. [CrossRef]

7. Jaremków, A.; Szałata, Ł.; Kołwzan, B.; Sówka, I.; Zwoździak, J.; Pawlas, K. Impact of a Sewage Treatment Plant on Health of Local Residents: Gastrointestinal System Symptoms. Pol. J. Environ. Stud. 2017, 26, 127-136. [CrossRef]

8. Uhrbrand, K.; Schultz, A.C.; Madsen, A.M. Exposure to airborne noroviruses and other bioaerosol components at a wastewater treatment plant in Denmark. Food Environ. Virol. 2011, 3, 130-137. [CrossRef]

9. Douglas, P.; Robertson, S.; Gay, R.; Hansell, A.L.; Gant, T.W. A systematic review of the public health risks of bioaerosols from intensive farming. Int. J. Hyg. Environ. Health 2018, 221, 134-173. [CrossRef] [PubMed]

10. Hu, Y.; Cheng, H.; Tao, S. Environmental and human health challenges of industrial livestock and poultry farming in China and their mitigation. Environ. Int. 2017, 107, 111-130. [CrossRef] [PubMed]

11. Garcia-Alcega, S.; Nasir, Z.A.; Ferguson, R.; Whitby, C.; Dumbrell, A.J.; Colbeck, I.; Gomes, D.; Tyrrel, S.; Coulon, F. Fingerprinting outdoor air environment using microbial volatile organic compounds (MVOCs)-A review. TrAC Trends Anal. Chem. 2017, 86, 75-83. [CrossRef]

12. Yoo, K.; Lee, T.K.; Choi, E.J.; Yang, J.; Shukla, S.K.; Hwang, S.; Park, J. Molecular approaches for the detection and monitoring of microbial communities in bioaerosols: A review. J. Environ. Sci. 2017, 51, $234-247$. [CrossRef] [PubMed]

13. O'Connor, D.J.; Daly, S.M.; Sodeau, J.R. On-line monitoring of airborne bioaerosols released from a composting/green waste site. Waste Manag. 2015, 42, 23-30. [CrossRef] [PubMed]

14. Stagg, S.; Bowry, A.; Kelsey, A.; Crook, B. Bioaerosol Emissions from Waste Composting and the Potential for Workers' Exposure. Health and Safety Laboratory; Research Report RR786; Health Safety Executive: Buxton, UK, 2010.

15. Breum, N.O.; Nielsen, B.H.; Nielsen, E.M.; Midtgaard, U.; Poulsen, O.M. Dustiness of compostable waste: A methodological approach to quantify the potential of waste to generate airborne micro-organisms and endotoxin. Waste Manag. Res. 1997, 15, 169-187. [CrossRef]

16. Heldal, K.K.; Breum, N.O.; Nielsen, B.H.; Wilkins, K. Experimental generation of organic dust from compostable household waste. Waste Manag. Res. 2001, 19, 98-107. [CrossRef] [PubMed]

17. Upton, S.L.; Mark, D.; Douglass, E.J.; Hall, D.J.; Griffiths, W.D. A wind tunnel evaluation of the physical sampling efficiencies of three bioaerosol samplers. J. Aerosol Sci. 1994, 25, 1493-1501. [CrossRef]

18. Andersen, A.A. New sampler for the collection, sizing, and enumeration of viable airborne particles. J. Bacteriol. 1958, 76, 471-484. [PubMed]

19. Anonymus. Water Quality-Detection and Enumeration of Legionella; ISO 11731; International Organisation for Standardisation: Geneva, Switzerland, 1998.

20. Bruker Daltonics. Inactivated Mycobacteria Bead Preparation Method (in Mbpm); Bruker Daltonics: Billerica, MA, USA, 2012. 
21. Currie, S.L.; Beattie, T.K.; Knapp, C.W.; Lindsay, D.S.J. Legionella spp. in UK composts-A potential public health issue? Clin. Microbiol. Infect. 2014, 20, O224-O229. [CrossRef] [PubMed]

22. Collins, S.; Jorgensen, F.; Willis, C.; Walker, J. Real-time PCR to supplement gold-standard culture-based detection of Legionella in environmental samples. J. Appl. Microbiol. 2015, 119, 1158-1169. [CrossRef] [PubMed]

23. Hernandez, M.; Perring, A.E.; McCabe, K.; Kok, G.; Granger, G.; Baumgardner, D. Chamber catalogues of optical and fluorescent signatures distinguish bioaerosol classes. Atmos. Meas. Tech. 2016, 9, 3283-3292. [CrossRef]

24. Pöhlker, C.; Huffman, J.A.; Pöschl, U. Autofluorescence of atmospheric bioaerosols-fluorescent biomolecules and potential interferences. Atmos. Meas. Tech. 2012, 5, 37-71. [CrossRef]

25. Pan, Y.-L.; Hill, S.C.; Pinnick, R.G.; Huang, H.; Bottiger, J.R.; Chang, R.K. Fluorescence spectra of atmospheric aerosol particles measured using one or two excitation wavelengths: Comparison of classification schemes employing different emission and scattering results. Opt. Express 2010, 18, 12436-12457. [CrossRef] [PubMed]

26. Hill, S.C.; Mayo, M.W.; Chang, R.K. Fluorescence of Bacteria, Pollens, and Naturally Occurring Airborne Particles: Excitation/Emission Spectra; Army Research Laboratory Computational and Information Sciences Directorate: Adelphi, MD, USA, 2009.

27. Savage, N.J.; Krentz, C.E.; Könemann, T.; Han, T.T.; Mainelis, G.; Pöhlker, C.; Huffman, J.A. Systematic characterization and fluorescence threshold strategies for the wideband integrated bioaerosol sensor (WIBS) using size-resolved biological and interfering particles. Atmos. Meas. Tech. 2017, 10, 4279-4302. [CrossRef]

28. Savage, N.; Huffman, J.A. Evaluation of a Hierarchical Agglomerative Clustering Method Applied to WIBS Laboratory Data for Improved Discrimination of Biological Particles by Comparing Data Preparation Techniques. Atmos. Meas. Tech. 2018, 11, 4929-4942. [CrossRef]

29. Pan, Y.-L. Detection and characterization of biological and other organic-carbon aerosol particles in atmosphere using fluorescence. J. Quant. Spectrosc. Radiat. Transf. 2015, 150, 12-35. [CrossRef]

30. Liebers, V.; Brüning, T.; Raulf-Heimsoth, M. Occupational endotoxin-exposure and possible health effects on humans. Am. J. Ind. Med. 2006, 49, 474-491. [CrossRef] [PubMed]

31. Traversi, D.; Alessandria, L.; Schiliro, T.; Gilli, G. Size-fractionated PM10 monitoring in relation to the contribution of endotoxins in different polluted areas. Atmos. Environ. 2011, 45, 3515-3521. [CrossRef]

32. Bartram, J.; Chartier, Y.; Lee, J.V.; Pond, K.; Surman-Lee, S. Legionella and the Prevention of Legionellosis; World Health Organization: Geneva, Switzerland, 2007.

33. Casati, S.; Conza, L.; Bruin, J.; Gaia, V. Compost facilities as a reservoir of Legionella pneumophila and other Legionella species. Clin. Microbiol. Infect. 2010, 16, 945-947. [CrossRef] [PubMed] 